\title{
The histopathology of non-steroidal anti-inflammatory drug induced gastroduodenal damage: correlation with Helicobacter pylori, ulcers, and haemorrhagic events
}

\author{
M Frezza, N Gorji, M Melato
}

\begin{abstract}
Aims-The spectrum of microscopic lesions resulting from the chronic use of non-steroidal anti-inflammatory drugs (NSAIDs), known as chemical gastritis, remains unclear, and the variable prevalence reported in different studies makes this issue a matter of lively debate. The aim of this study was to evaluate the prevalence and importance of chemical gastritis in patients regularly taking NSAIDs. Owing to the high prevalence of Helicobacter pylori infection, particularly in subjects over 60 years of age, and in view of a possible association with damage, the presence of $H$ pylori infection in the same tissue sample was also determined in all patients.
\end{abstract}

Methods-One hundred and ninety seven subjects were enrolled, 118 of whom were receiving chronic treatment with NSAIDs and 79 of whom were controls, pair matched for age, sex, and clinical symptoms (ulcer-like dyspepsia or upper digestive tract haemorrhage). Antral biopsies taken during upper gastroduodenal endoscopy were assessed for chemical gastritis according to a modified version of Dixon's score, and for helicobacter correlated chronic active gastritis, according to the updated Sydney system.

Results-Chemical gastritis was identified in 11 patients taking NSAIDs (9\%) and in four controls $(5 \%) \quad(p<0.05)$. Helicobacter pylori was detected in 53 patients taking NSAIDs (45\%) and in 34 controls $(43 \%)$. Patients taking NSAIDs had a significantly higher number of erosions and ulcers and worse endoscores than controls. The presence of $H$ pylori did not appear to increase histological damage, ulcer prevalence, or haemorrhagic events.

Conclusions-Chemical gastritis is present in a limited number of patients regularly taking NSAIDs, and is not strongly correlated with NSAID induced damage. In many cases of peptic ulcer or upper gastrointestinal bleeding in patients taking NSAIDs, the presence of chemical gastritis or $H$ pylori infection cannot solely account for the development of mucosal damage.

(f Clin Pathol 2001;54:521-525)

Keywords: chemical gastritis; non-steroidal anti-inflammatory drugs; Helicobacter pylori
The chronic use of non-steroidal antiinflammatory drugs (NSAIDs) is a common cause of gastroduodenal erosions and peptic ulcers resulting, in many cases, in fatal haemorrhage. ${ }^{1-10}$

NSAIDs and Helicobacter pylori infection are thought to be the two most important exogenous factors in gastric and duodenal ulcer disease. The relative frequency of $H$ pylor $i$ and NSAIDs as the cause of peptic ulcers varies and is, at least in part, correlated to the prevalence of $H$ pylori infection and the use of NSAIDs in a population, ${ }^{11}$ as well as to the expertise in identifying the presence of $\mathrm{H}$ pylori infection. ${ }^{12}$

The clinical and demographic data of NSAIDs and $H$ pylori related gastroduodenal ulcers are considerably different. Whereas the histopathology of $H$ pylori correlated gastritis is associated with well defined histological features, ${ }^{13}$ the spectrum of microscopic gastric lesions caused by chronic ingestion of NSAIDs is unspecific and is currently a matter of lively debate. ${ }^{14-18}$

Moreover, because NSAIDs are widely used in the elderly ${ }^{19}$ and the incidence of $H$ pylori infection is increased in the elderly ${ }^{20} \mathrm{H}$ pylori should be present in almost $50 \%$ of those patients over 60 years taking NSAIDs.

Although the damage mechanisms of these two factors probably follow independent pathways ${ }^{21-23}$ from a practical point of view it is important to establish whether their effects are the sum of two independent forms of injury (additive effect) or whether one form of injury can enhance the effect of the other (synergistic effect). Similarly, it is crucial to determine whether we should diagnose and cure $H$ pylori infection in all patients about to undergo long term treatment with NSAIDs.

The aim of our study was to define the histological spectrum of the gastric damage seen in symptomatic patients who are regular users of NSAIDs and its relation to $H$ pylori infection, endoscopic lesions (erosions and/or ulcers), and a major clinical event such as haemorrhage.

\section{Patients and methods}

One hundred and eighteen adult patients treated regularly with NSAIDs for a variety of conditions and observed in our unit for signs of potential injury to the gastroduodenal mucosae (such as ulcer-like dyspepsia or upper intestinal tract haemorrhage) were examined by upper gastroduodenal endoscopy. All the patients
Accepted for publication 16 January 2001 
with haemorrhage presented melena or haematemesis of mild or medium severity; to be enrolled, however, they had to be able to undergo the endoscopic examination. The drugs, which had been used for at least one month at the dosage indicated, were: aspirin (100-200 mg), diclofenac (100-200 mg), naproxen (500-1000 mg), and ketoprofen (100-200 mg/day) in 68, 22, nine, and 19 patients, respectively. Seventy nine subjects not regularly taking NSAIDs, pair matched for age, sex, race, and symptoms, were used as controls. The exclusion criteria were: recent use of antibiotics, antisecretory (particularly proton pump inhibitors) or cytostatic drugs, and previous gastroduodenal surgery. All patients were asked for complete clinical notes and were questioned about habits such as smoking and alcohol intake. Informed consent was obtained before the upper gastroduodenal endoscopy, which was performed without medication.

The endoscopic lesions were graded according to the following score ${ }^{14}: 0$, minor erythema; 1 , antral or duodenal erosions $(\leqslant 5)$; 2 , antral and duodenal erosions (or $>5$ ); 3 , ulcerations measuring $5 \mathrm{~mm}$ in diameter or more.

A set of two to four mapped antrum biopsy specimens was obtained from patients and controls. Histology of all biopsies, processed routinely and stained by haematoxylin and eosin, according to the method of MayGrünwald-Giemsa, was assessed independently and blindly by two different pathologists trained in gastrointestinal pathology, and a third pathologist was consulted in the event of disagreement.

Chronic inflammation (CI), $H$ pylori density (HpD), polymorphonuclear neutrophil activity (PNA), glandular atrophy (GA), and intestinal metaplasia (IM) were classified according to the updated Sydney system. ${ }^{24}$ To improve comparability of parameters, specimens were scored for $\mathrm{HpD}$, IM, and GA as positive (1) or negative (0); CI and PNA were graded as negative (0), mild (1), moderate (2), or pronounced (3). Patients scoring 4 or more were considered to have chronic active gastritis. This form is characterised by a high infiltration of polymorphonuclear neutrophils (PMN) attracted to the tissue by the presence of $H p y$ lori (fig 1).

All biopsies were also graded according to a modified version of Dixon's system for chemical gastritis. ${ }^{25}$ Foveolar hyperplasia, oedema, and prominent smooth muscle fibres were graded as absent ( 0 ) or present (1) because, in our experience, a more extensive score does not work; loss of PMN and plasma cells were scored inversely from many (0) to absent (3). Vasodilatation and congestion were not considered because, in our view, they are mostly the result of bioptic trauma and are therefore also present in patients from the control group. Patients scoring more than 6 were considered to have chemical gastritis (fig 2).

The data were analysed using the $\chi^{2}$ test for the per cent values and the Student's paired $t$ test for the comparison of mean values; $p$

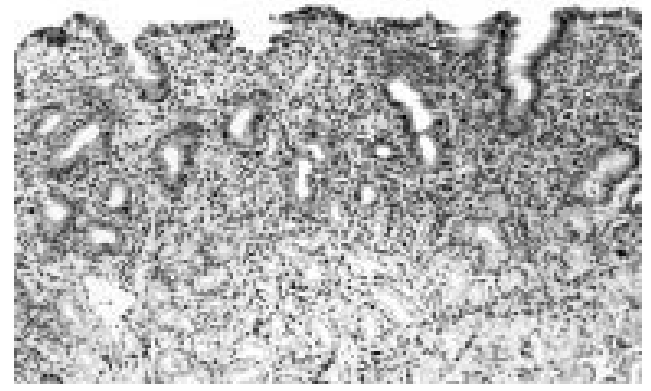

Figure 1 A case of helicobacter correlated chronic active gastritis with evident infiltration of polymorphonuclear neutrophils (haematoxylin and eosin stained).

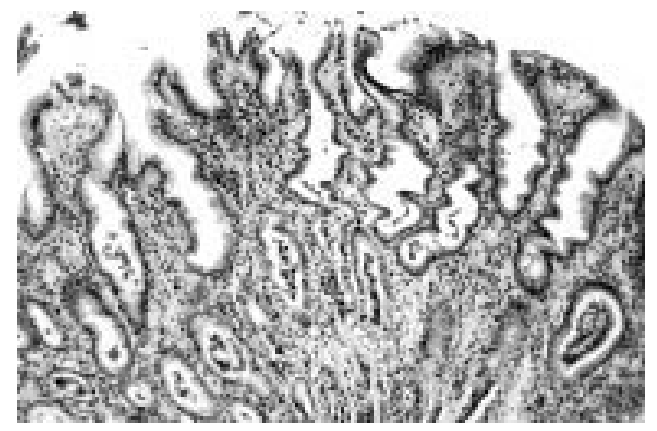

Figure 2 A case of chemical gastritis showing absence of polymorphonuclear neutrophils and evident foveolar hyperplasia (haematoxylin and eosin stained).

values $<0.05$ were considered significant. Statistical analysis was performed with the SPSS for Windows Release 7.1 (Microsoft).

\section{Results}

A total of 197 subjects were examined, 118 of whom were regular NSAID users, and 79 of whom were controls. Table 1 gives the demographic and clinical details of the patients and controls.

Table 2 details the histological pattern of the antral mucosa seen in the two groups. Chemical gastritis was only present in a minority of patients using NSAIDs, but its prevalence was

Table 1 Demographic and clinical details of patients and controls

\begin{tabular}{lll}
\hline & $\begin{array}{l}\text { NSAID users } \\
(n=118)\end{array}$ & $\begin{array}{l}\text { Controls } \\
(n=79)\end{array}$ \\
\hline Sex $(\mathrm{M} / \mathrm{F})$ & $51 / 67$ & $31 / 48$ \\
Mean age (range), years & $66.5(22-88)$ & $67.3(21-87)$ \\
Smokers & 40 & 18 \\
Alcoholics & No & No \\
Signs of potential gastroduodenal mucosal injury \\
Ulcer-like dyspepsia & 94 & 63 \\
Haemorrhages & 24 & 16 \\
\hline
\end{tabular}

NSAID, non-steroidal anti-inflammatory drugs.

Table 2 Histological pattern of antral mucosa in the two groups

\begin{tabular}{lclc}
\hline & $\begin{array}{l}\text { NSAID users } \\
(n=118)\end{array}$ & $p$ Value & $\begin{array}{c}\text { Controls } \\
(n=79)\end{array}$ \\
\hline Chemical gastritis & $11(9 \%)$ & $<0.05$ & $4(5 \%)$ \\
Active gastritis & $34(29 \%)$ & NS & $15(19 \%)$ \\
Chronic gastritis & $64(54 \%)$ & NS & $53(67 \%)$ \\
No lesions & $9(8 \%)$ & NS & $7(9 \%)$ \\
Helicobacter pylori & $53(45 \%)$ & NS & $32(41 \%)$ \\
\hline
\end{tabular}

Significance was calculated by means of the $\chi^{2}$ test. NS, not significant; NSAID, non-steroidal anti-inflammatory drugs. 
Table 3 Endoscopic and histological findings in NSAID users and controls complaining of ulcer-like dyspepsia

\begin{tabular}{|c|c|c|c|c|}
\hline \multirow[b]{2}{*}{ Erosions } & $\begin{array}{l}\text { NSAID users } \\
(n=94)\end{array}$ & \multirow{2}{*}{$\begin{array}{l}\text { p Value } \\
<0.05\end{array}$} & \multicolumn{2}{|c|}{$\begin{array}{l}\text { Controls } \\
(n=63)\end{array}$} \\
\hline & $(35 \%)$ & & 15 & $(24 \%)$ \\
\hline Ulcers & $(23 \%)$ & $<0.001$ & 4 & $(6 \%)$ \\
\hline Mean endoscore (SD) & $1.20(0.48)$ & $<0.05$ & 0.46 & $(0.21)$ \\
\hline Chemical gastritis & $(8 \%)$ & NS & 3 & $(5 \%)$ \\
\hline Active gastritis & $(21 \%)$ & NS & 12 & $(19 \%)$ \\
\hline Helicobacter pylori & $(48 \%)$ & NS & 26 & $(41 \%)$ \\
\hline
\end{tabular}

Significance was calculated by means of the Student's $t$ test for mean values and the $\chi^{2}$ test for percentages.

NS, not significant; NSAID, non-steroidal anti-inflammatory drugs.

Table 4 Endoscopic and histological findings in NSAID users according to Helicobacter pylori infection

\begin{tabular}{|c|c|c|c|c|c|}
\hline \multirow[b]{2}{*}{ Age (years) } & \multicolumn{3}{|c|}{$\begin{array}{l}\text { Helicobacter present } \\
(n=53)\end{array}$} & \multicolumn{2}{|c|}{$\begin{array}{l}\text { Helicobacter absent } \\
(n=65)\end{array}$} \\
\hline & 67.3 & $(40-84)$ & & 66.1 & $(22-88)$ \\
\hline Erosions & 23 & $(43 \%)$ & NS & 24 & $(37 \%)$ \\
\hline Ulcers & 17 & $(32 \%)$ & NS & 21 & $(32 \%)$ \\
\hline Mean endoscore (SD) & 1.28 & $(0.38)$ & NS & 1.27 & $(0.21)$ \\
\hline Chemical gastritis & 0 & $(0 \%)$ & - & 11 & $(17 \%)$ \\
\hline Active gastritis & 17 & $(32 \%)$ & $\mathrm{p}<0.03$ & 7 & $(11 \%)$ \\
\hline
\end{tabular}

Significance is calculated by means of the Student's $t$ test for means and $\chi^{2}$ for percentages. NS, not significant; NSAID, non-steroidal anti-inflammatory drug.

higher than in controls. The other types of gastritis (chronic active gastritis and chronic nonactive gastritis, or, strictly speaking, chronic gastritis) were equally distributed across the two groups, with the most common form being chronic non-active gastritis. The prevalence of H pylori $(\sim 40-50 \%)$ was not significantly different in the two groups.

Of the patients complaining of ulcer-like dyspepsia (table 3), the group of NSAID users had significantly more endoscopic lesions (erosions and/or ulcers), although the percentage of chemical or active gastritis did not reach significance. The number of patients infected with $H$ pylori was similar in the two groups $(48 \%$ v $41 \%)$.

With regard to $H$ pylori in the NSAID user group (table 4), the 53 H pylori positive patients had similar endoscopic patterns to the $65 \mathrm{H}$ pylori negative patients, as well as a higher percentage of active gastritis and no cases of chemical gastritis.

A total of 38 ulcers-21 duodenal, 15 gastric, and two gastric and duodenal-were diagnosed in the patients taking NSAIDs, 17 of whom were $H$ pylori positive (seven gastric and 12 duodenal ulcers). Within this group, the distribution of chemical and active gastritis was not significantly different between the patients with and without ulcers, whereas the number of erosions was higher in the last subgroup (table 5).

Table 5 Endoscopic and histological findings in NSAID users according to the presence of ulcers and haemorrhagic events

\begin{tabular}{lcllr}
\hline Patients & Erosions & $\begin{array}{l}\text { Mean } \\
\text { endoscore (SD) }\end{array}$ & $\begin{array}{l}\text { Chemical } \\
\text { gastritis }\end{array}$ & $\begin{array}{l}\text { Helicobacter } \\
\text { pylori }\end{array}$ \\
\hline Ulcers $(\mathrm{n}=38)$ & $6(16 \%)$ & $3.0(0.0)$ & $3(8 \%)$ & $17(45 \%)$ \\
No ulcers $(\mathrm{n}=80)$ & $40(49 \%)^{\star}$ & $0.57(0.31)^{\star}$ & $7(9 \%)$ & $36(45 \%)$ \\
Haemorrhages $(\mathrm{n}=24)$ & $10(42 \%)$ & $2.41(0.51)$ & $3(12 \%)$ & $8(33 \%)$ \\
No haemorrhages $(\mathrm{n}=94)$ & $33(35 \%)$ & $1.20(0.48) \dagger$ & $8(8 \%)$ & $45(48 \%)$ \\
\hline
\end{tabular}

Significance was calculated by means of the Student's $t$ test for means and $\chi^{2}$ test for percentages.

${ }^{\star} \mathrm{p}<0.01$ between patients with and without ulcers.

$t \mathrm{p}<0.05$ between patients with and without haemorrhages.

NSAID, non-steroidal anti-inflammatory drug.
Patients in the control group had a total of 12 ulcers - nine duodenal, two gastric, and one gastric and duodenal-and only six of these patients were positive for $H$ pylori infection.

NSAID users complaining of one or more haemorrhagic episodes had more ulcers and worse endoscores than the others, although the prevalence of chemical and active gastritis did not reach significance. The prevalence of $H$ pylori was considerably, although not significantly, lower (33\% v 48\%) than in those patients without haemorrhage (table 5).

Finally, all the NSAIDs used in our series had a low damaging profile, which might account for the fact that we did not find a significant correlation between the severity of the endoscopic and/or histological finding and the type of drug used.

\section{Discussion}

In 1986, Dixon et al investigated several morphological parameters of the gastric mucosa (foveolar hyperplasia, occurrence of smooth muscle fibres and oedema in the lamina propria, vasodilatation, congestion of superficial capillaries, and paucity of both acute and chronic inflammatory cells) and found a strong association between severe grades of each of these histological variables and both hypochlorhydria and increased bile acid concentrations in the stomach, suggesting that reflux gastritis was a distinct histopathological entity. ${ }^{25} \mathrm{~A}$ few years later, Sobala et al stated that most cases of so called "reflux gastritis" in the intact stomach did not result from the reflux of bile, but were caused by NSAID use, and suggested calling this type of gastritis "C" or "chemical". ${ }^{26}$ In 1992, Laine et al considered a different set of morphological parameters and concluded that the development of ulcers (but not gastritis) in patients taking NSAIDs did not require $H$ pylori. ${ }^{27}$

Caselli et al reported that distinguishing features in patients treated with NSAIDs were erosions, macroerosions, and the presence of prominent capillaries in suberosive areas of the lamina propria. ${ }^{16}$ Finally, El-Zimaity et al concluded that none of these histological features could be used singularly to characterise the diagnosis of chemical gastropathy. ${ }^{15}$

The variability of the data reported in the literature may have a variety of explanations, the most plausible being the fact that the parameters used to define chemical gastritis are not well defined. Foveolar hyperplasia, which appears to be a result of excessive cell exfoliation from the surface epithelium or of stimulation by cytokines or other inflammatory mediators, was first reported in patients with bile reflux ${ }^{28} 29$ and subsequently observed after gastric surgery ${ }^{25}$ in long term NSAID users. ${ }^{26}$ Currently, according to the updated Sydney system, ${ }^{24}$ it "may be seen in all forms of gastritis, but is most pronounced in chemical gastritis". Oedema, vasodilatation, haemorrhage, and telangiectasia are unstable features that are possibly related to local factors and/or biopsy. The increased number of smooth muscle fibres in the lamina propria was originally demonstrated by Dixon and colleagues ${ }^{25}$ but, although 
usually reported as characteristic of chemical gastritis, in later studies it was not seen in long term NSAID users with chemical gastritis ${ }^{26}$ or in chemical gastritis induced by naproxen. ${ }^{17}$ Erosion and macroerosion are not often present in biopsy specimens because they are dependent on the sectioning and localisation of the endoscopic biopsy.

Nonetheless, the prevalence of chemical gastritis in chronic NSAID users, described by the different authors, varies between $26 \%{ }^{14}$ and $45 \% .{ }^{17}$ In our study, we calculated a prevalence of about $10 \%$ in the whole population studied (table 2), with prevalence being only slightly lower among those patients with ulcers and not significantly higher in those reporting digestive haemorrhage (table 5). As can be seen in table 2 , four patients in the control group had scores consistent with chemical gastritis. These patients had not taken NSAIDs in the three months preceding the study, and neither were they alcohol abusers. However, it cannot be excluded that these patients had taken other medications or ingested foods that were potentially harmful to the mucosa. In statistical terms they have to be considered false positives. These data confirm, in our view, that chemical gastritis is not a pathognomonic histotype of the chronic gastric damage caused by the use of NSAIDs. According to El-Zimaity and colleagues ${ }^{15}$ this may be accounted for by a variety of reasons, including the facts that of all the patients regularly taking NSAIDs, only a few (those with greater sensitivity) develop chemical gastritis, and mucosal damage can be patchy rather than uniform.

Other findings of our study are also worthy of note. The first is the relation between chronic NSAID use and colonisation by $H p y$ lori. The first papers on this question reported a lower prevalence of $H$ pylori in the gastric mucosa of patients receiving chronic NSAID treatment. ${ }^{14162730}$ It was thought that the gastric environment created by the use of antiinflammatory medication might be unfavourable to $H$ pylori implantation, ${ }^{14}$ a view confirmed by the fact that NSAIDs can block the growth of the bacterium in vitro. ${ }^{31}$

Later, possibly because of improved accuracy of diagnostic methods, other studies reported an equal prevalence of $H$ pylori in chronic NSAID users and control groups..$^{15} 172$

Similarly, the prevalence of $H$ pylori seen in our study $(\sim 45-50 \%)$ was similar in the two groups, leading us to exclude the possibility that anti-inflammatory drugs can impair implantation and growth of $\mathrm{H}$ pylori in the stomach mucosa. Thus, we can conclude that bacteria and medications are two frequent but independent aetiological damaging factors.

Another much debated topic is whether the presence of $H$ pylori can modify the histological picture associated with NSAID induced damage and, if so, in what way. According to some authors, chemical gastritis is only prevalent where $H$ pylori infection is absent. ${ }^{14}{ }^{17}$ This may be because the inflammation caused by the infection alters the characteristics of the pharmacological damage that has partly reverse histological features. In Dixon's scoring system, ${ }^{25}$ chemical gastritis and chronic active gastritis are in fact mutually exclusive. Even El-Zimaity et al concluded that simultaneous infection by $H$ pylori makes the histological diagnosis of chemical gastritis extremely difficult. ${ }^{15}$ In agreement with these data, none of our $53 \mathrm{H}$ pylori positive patients who were chronic users of NSAIDs had chemical gastritis, whereas about a third of these patients had a score consistent with active chronic gastritis.

NSAIDs can cause considerable damage and are associated with a high prevalence of gastroduodenal ulcers. NSAID related ulcers might originate by two possible mechanisms: the concomitant presence of $H$ pylori infection and/or the mucosal damage that was thought to show up as chemical gastritis. ${ }^{24}$ Laine et al have already shown that gastric ulcers associated with the use of NSAIDs are an important subgroup of ulcers that do not require the presence of $H$ pylori infection for their formation. ${ }^{27}$

In our series of patients taking NSAIDs, $H$ pylori infection was invariably close to $45 \%$, both in those with ulcers and in those without (table 5). Of the 38 patients with ulcers, 17 were $H$ pylori positive and three had chemical gastritis, but in 18 neither of these factors could explain the presence of the ulcer.

It must be concluded that, at least in our cases, the presence of $H$ pylori does not seem to enhance the risk of the development of ulcers, so that in many cases NSAIDs might be responsible for producing the lesions via different pathways to those mentioned above. NSAIDs also act by means of other damage mechanisms, the most important being inhibition of the synthesis of prostaglandins ${ }^{33}$ and functional rupture of the mucosal barrier. ${ }^{34}$ These factors may underlie erosion and/or ulcers in $H$ pylori negative cases not presenting evidence of chemical gastritis.

Even in patients taking NSAIDs it is still unclear whether concomitant infection with $H$ pylori plays a synergic role in causing bleeding. This hypothesis has been supported by some authors, ${ }^{35-37}$ but not confirmed by others. $^{38-41}$ One paper has even shown that the presence of $H$ pylori significantly prevents the depletion of tissue prostaglandins caused by NSAIDs, thus reducing their damage potential. ${ }^{42}$

In our 24 chronic users of NSAIDs who reported one or more episode of upper intestinal tract haemorrhage, the bleeding originated from a gastric ulcer in 10 patients, a duodenal ulcer in four, a haemorrhagic gastropathy with erosions in eight, and a duodenal ulcer with gastric erosions in two. In this group, in agreement with the literature data that report a low prevalence of $H$ pylori in patients with haemorrhage, ${ }^{43}$ we identified $H$ pylori infection in only eight cases; three other cases had chemical gastritis. In 13 patients, none of whom were alcoholics, the mechanism underlying the mucosal damage was not easily identifiable on the basis of the histological findings (table 5).

These results seem to indicate that, as was noted for ulcers, the presence of $H$ pylori does not increase the prevalence of haemorrhage. 
In conclusion, our study has shown that neither chemical gastritis nor the presence of $H$ pylori strongly correlates with the gastroduodenal damage induced by NSAIDs. This damage eludes all attempts at histological characterisation: the histological parameters put forward to date lack sufficient sensitivity or specificity to be considered reliable.

1 Lanza FL. A review of gastric ulcer and gastroduodenal injury in normal volunteers receiving aspirin and other nonsteroidal anti-inflammatory drugs. Scand $\mathcal{7}$ Gastroenterol 1989;24(suppl 163):24-31.

2 CMS update: Non-steroidal anti-inflammatory drugs and serious $1190-1$.

3 Clinich D, Sanergee AK, Ostick G. Non-steroidal antiinflammatory drugs and gastrointestinal adverse effects. $\mathcal{F}$ $R$ Coll Physicians Lond 1983;17:228-30.

4 Collier DSTJ, Pain JA. Non-steroidal anti-inflammatory Collier DSTJ, Pain JA. Non-steroidal anti-inflammatory
drugs and peptic ulcer perforation. Gut 1985;26:359-63.

drugs and peptic ulcer perforation. Gut 1985;26:359-63.
5 Llewelyn IG, Pritchard MN. Acute gastric haemorrhage and its relationship to the use of anti-inflammatory analgesics its relationship to the use of anti-inflammator
(NSAIDs). Ann Rheum Dis 1983;42:228-9.

6 Alexander AM, Veitch GSA, Wood JS. Anti-rheumatic and analgesic drug usage and acute gastrointestinal bleeding in elderly patients. F Clin Hosp Pharm 1985;10:89-93.

7 Armstrong GP, Blower AL. Non-steroidal antiinflammatory drugs and life-threatening complications of peptic ulceration. Gut 1987;28:527-32.

8 Jolobe OMP, Montgomery RD. Changing clinical pattern of gastric ulcer: are anti-inflammatory drugs involved? Digestion 1984;29:164-70.

9 Somerville K, Faulkner G, Langman MJS. Non-steroidal anti-inflammatory drugs and bleeding peptic ulcer. Lancet 1986;1:462-4.

10 Graham DY, Smith JL. Gastroduodenal complications of chronic NSAID therapy. Am f Gastroenterol 1988;83:10814 .

11 Al-Assi MT, Genta RM, Karttunen TJ, et al. Ulcer site and complications: relation to $\mathrm{Helicobacter}$ pylori infection and complications: relation to Helicobacter pylor

12 Genta RM, Robason GO, Graham DY. Simultaneous visualization of Helicobacter pylori and gastric morphology: alization of Helicobacter pylori and ga

13 Whitehead R, Truelove SC, Gear MWL. The histological diagnosis of chronic gastritis in fibreoptic gastroscope biopsy specimens. F Clin Pathol 1972;25:1-11

14 Taha AS, Nakshabendi I, Lee FD, et al. Chemical gastritis and Helicobacter pylori related gastritis in patients receiving non-steroidal anti-inflammatory drugs: comparison and correlation with peptic ulceration. $f$ Clin Pathol 1992;45:135-9.

15 El-Zimaity HMT, Genta RM, Graham DY. Histological features do not define NSAID-induced gastritis. Hum Patho 1996;27:1348-54.

16 Caselli M, La Corte R, De Carlo L, et al. Histological findings in gastric mucosa in patients treated with non-steroidal ings in gastric mucosa in patients treated with non-steroidal

17 McCarthy CJ, McDermott M, Hourihane D, et al. Chemical gastritis induced by naproxen in the absence of Helicocal gastritis induced by naproxen in the absence of
bacter pylori infection. $\mathcal{F}$ Clin Pathol $1995 ; 48: 61-3$. 18 Shah K, Price AB, Talbot IC, et al. Effect of longterm misoinflammatory drugs: a histological study. Gut 1995;37:1958.

19 Committee on Safety of Medicines. Non-steroidal antiinflammatory drugs and serious gastrointestinal adverse reactions. BMF 1986;292:614-15.

20 Graham DY, Lidsky MD, Cox AM, et al. Long-term non-steroidal anti-inflammatory drug use and Helicobacter pylori infection. Gastroenterology 1991;100:1653-7.

21 Malfertheiner P, Labenz J. La presenza di Helicobacter pylori influenza la patologia gastroduodenale associata ai farmaci anti-inflammatori non steroidei? $A m$ f Med 1998 [Ed. italiana];104:37-43.

22 Vane JR. Inhibition of prostaglandin synthesis as a mechanism of action for aspirin-like drugs. Nature New Biology 1971;231:232-5.

23 Crabtree JE. Gastric mucosal inflammatory responses to Helicobacter pylori. Aliment Pharmacol Ther 1996;10(suppl 1):29-37.

24 Dixon MF, Genta RM, Yardley JH, et al. Classification and grading of gastritis. The updated Sydney system. Am f Surg Pathol 1996;20:1161-81.

25 Dixon MF, O'Connor HJ, Axon ATR, et al. Reflux gastritis: distinct histopathological entity? f Clin Pathol 1986;39: $524-30$

26 Sobala GM, King RFG, Axon ATR, et al. Reflux gastritis in the intact stomach. F Clin Pathol 1990;43:303-6.

27 Laine L, Marin-Sorensen M, Weinstein WM. Nonsteroidal anti-inflammatory drug-associated gastric ulcers do not require Helicobacter pylori for their development. Am $\mathcal{F}$ Gastroenterol 1992;87:1398-402.

28 Dewar EP, Dixon MF, Johnston D. Bile reflux and degree of gastritis after highly selective vagotomy, truncal vagotomy, and partial gastrectomy for duodenal ulcer. World $\mathcal{F}$ Surg 1983;7:743-50.

29 Dewar EP, Dixon MF, Johnston D. Bile reflux and degree of gastritis in patients with gastric ulcer: before and after operation. F Surg Res 1984;37:277-84.

30 Talley NJ, Evans JM, Fleming KC, et al. Nonsteroidal antiinflammatory drugs and dyspepsia in the elderly. Dig Dis Sci 1995;40:1345-50.

31 Caselli M, Pazzi P, La Corte R, et al. Campylobacter-like organism nonsteroidal anti-inflammatory drugs and gastric lesion in patients with rheumatoid arthritis. Digestion 1989; 44:101-4.

32 Santucci L, Fiorucci S, Patoia L, et al. Severe gastric mucosal damage induced by NSAIDs in healthy subjects is associated with Helicobacter pylori infection and high levels of serum pepsinogens. Dig Dis Sci 1995;40:2074-80

33 Whittle BJR, Higgs GA, Eakins KE, et al. Selective inhibition of prostaglandin production in inflammatory exudates and gastric mucosa. Nature 1980;284:271-3.

34 Cooke AR. The role of the mucosal barrier in drug-induced gastric ulceration and erosions. Dig Dis Sci 1976;21:15564

35 Russell RI. Helicobacter pylori eradication may reduce the risk of gastroduodenal lesions in chronic NSAID users. Ital f Gastroenterol Hepatol 1997;29:465-9.

36 Taha AS, Russel RI. H. pylori and non-steroidal antiinflammatory drugs. Uncomfortable partners in peptic ulcer disease. Gut 1993;34:580-4.

37 Chan FK, Sung JJ, Chung SC, et al. Randomised trial of eradication of $\mathrm{H}$. pylori before non-steroidal antiinflammatory drug therapy to prevent peptic ulcers. Lancet 1997;350:975-9.

38 Yeomans ND, Tulassay Z, Juhasz L, et al. A comparison of omeprazole with Ranitidine for ulcers associated with nonsteroidal anti-inflammatory drugs. N Engl f Med 1998;338: $719-26$.

39 Hawkey CJ, Karrasch JA, Szczepanski L, et al. Omeprazole compared with misoprostol for ulcers associated with nonsteroidal anti-inflammatory drugs. N Engl F Med 1998;338: 727-34.

40 Hawkey CJ, Tulassay Z, Szczepanski L, et al. Randomised controlled trial of Helicobacter pylori eradication in patients on nonsteroidal anti-inflammatory drugs: HELP NSAIDs study, Lancet 1998;352:1016-21.

41 Wu CY, Poon SK, Chen GH, et al. Interaction between Helicobacter pylori and NSAID in peptic ulcer bleeding. Scand $\mathcal{f}$ Gastroenterol 1999;3:234-7.

42 Hudson N, Balsitis M, Filipowicz F, et al. Effect of Helicobacter pylori colonisation on gastric mucosal eicosanoid synthesis in patients taking NSAIDs. Gut 1993;34:748-51.

43 Bianchi Porro G, Lazzaroni $M$. The conflicting relationship between Helicobacter pylori and non-steroidal antiinflammatory drugs in peptic ulcer bleeding. Scand $\mathcal{F}$ Gastroenterol 1999;3:225-8. 